\title{
NEGATIVELY RICCI CURVED MANIFOLDS
}

\author{
JOACHIM LOHKAMP
}

\begin{abstract}
In this paper we announce the following result: "Every manifold of dimension $\geq 3$ admits a complete negatively Ricci curved metric." Furthermore we describe some sharper results and sketch proofs.
\end{abstract}

\section{INTRODUCTION}

There are three well-known curvatures: sectional, Ricci, and scalar curvature. While the existence of complete negatively sectional curved metrics leads to many topological implications for the underlying manifold, each manifold admits a complete metric of constant negative scalar curvature.

Since Ricci curvature takes position between these two curvatures, it is reasonable to look for obstructions as well as for existence results for (complete) negatively Ricci curved metrics.

This led to some long-standing conjectures in Riemannian geometry and appears in lists of problems compiled by Yau [Y], problem 24, Kazdan [K], problem 9, Bourguignon $[\mathrm{Bg}]$, question 4.11 , and others.

A first notable result was proved by Gao and Yau (cf. [G, GY]). They started from Thurston's Hyberbolic Dehn Surgery [T]: Every compact three-manifold $M$ can be obtained from $S^{3}$ by Dehn Surgery, along some link $L_{M} \subset S^{3}$, which complement $S^{3} \backslash L_{M}$, admits a complete hyperbolic metric with finite volume. Gao and Yau managed to perform this Dehn Surgery such that the Ricci curvature $r(g)$ remains negative near $L_{M}$ and is just the hyperbolic metric outside, i.e., one gets a metric with $r(g)<0$ on each compact threemanifold. Finally they extended this result to three-manifolds of finite type (with complete metric).

Subsequently, there was a paper, written by Brooks [Br], that addressed general existence theorems. He develops a technique of smoothing hyperbolic orbifold singularities of order $K \geq 12$, which could be locally realized as the quotient of hyperbolic space by an element of order $K$ fixing a codimension 2 manifold $N$ (which is hyperbolic if $\operatorname{dim} N \geq 2$ ).

On the other hand, Thurston theory yields such higher singular hyperbolic orbifold metrics on each compact three-manifold and Brooks combines these ideas to get: Every compact three-manifold admits a metric $g$ with $-a<$ $r(g)<-b$ for constants $a>b>0$, which are independent of the chosen manifold.

In contrast to that we will develop a completely different method of attack.

Received by the editors November 6, 1991.

1991 Mathematics Subject Classification. Primary 53C20, 57R99. 
Starting from an almost arbitrary metric, we obtain our result by local deformations, which will be sketched in part III of this paper.

\section{NEW RESULTS}

Theorem 1. There are constants $a(n)>b(n)>0$ depending only on the dimension $n \geq 3$, such that each manifold $M^{n}$ admits a complete metric $g$ with

$$
-a(n)<r(g)<-b(n) .
$$

Notice that even for $n=3$ we give a proof that does not use Thurston theory.

Bochner proved a classical result: The isometry group of a compact manifold with $r(g)<0$ is finite. We will show that this "geometric restriction" is sharp.

Theorem 2. If $M^{n}, n \geq 3$, is a compact manifold and $G$ is a subgroup of $\operatorname{Diff}(M)$, then

$$
G=\operatorname{Isom}(M, g) \text { for some metric } g \text { with } r(g)<0 \Leftrightarrow G \text { is finite. }
$$

Next, there are unexpected density and cut-off properties of negatively Riccicurved metrics.

Gromov [Gr] introduced the so-called Hausdorff distance $d_{\mathrm{H}}$ between two metric spaces $M_{1}, M_{2}$, which can be viewed as the minimal distance or deviation between $M_{1}$ and $M_{2}$ for all possible isometric embeddings in any metric space $M$. Of course, Riemannian manifolds can also be considered as metric spaces, and we get for compact $M^{n}, n \geq 3$,

Theorem 3. $\left\{\left(M^{n}, g\right) \mid r(g)<0\right\}$ is dense in $\left\{\left(M^{n}, g\right) \mid g\right.$ arbitrary metric $\}$ with respect to $d_{\mathrm{H}}$.

Our final result is the most flexible one. It is a useful tool for constructing negatively curved metrics.

Theorem 4. Let $S \subset M^{n}, n \geq 3$, be a closed subset, $S \subset U$ an open neighborhood, and $g_{0}$ a metric on $S$ with $r\left(g_{0}\right) \leq 0$ (resp. $r\left(g_{0}\right)<0$ ) on $U$. Then there is metric $g$ on $M$ with $r(g) \leq 0($ resp. $r(g)<0)$ on $M$, and $g \equiv g_{0}$ on $S$.

Remark. Theorem 1 implies that each manifold admits a complete metric with scalar curvature bounded by two negative constants. In this case, the Yamabe equation can be solved easily, and we get a complete metric of constant negative scalar curvature. This was proved before by Aubin [A] and Bland and Kalka $[B K]$ in a different way.

\section{SOME IDEAS OF THE PROOF}

The proof is by induction (with respect to dimension) and consists of two main parts:

(1) There is a metric $g_{n}^{-}$on $\mathbb{R}^{n}$ with $r\left(g_{n}^{-}\right)<0$ on $B_{1}(0)$ and $g_{n}^{-} \equiv g_{\text {Eucl. }}$ on $\mathbb{R}^{n} \backslash B_{1}(0)$.

(2) Using this metric $g_{n}^{-}$a Besicovitch type covering argument yields the theorems for $n$-dimensional manifolds.

In part (1) there are two cases where the proofs are completely different: $n=3$ and $n \geq 4$. 
The case $n=3$ starts from a metric $g$ on $\mathbb{R}^{3} \# S^{1} \times S^{2}$, which is isometric to $\left(\mathbb{R}^{3} \backslash B_{1}(0), g_{E u c l}\right.$. $)$ outside a set $U$, with $r(g)<0$ on $U$. By some warped product trick, which also allows to extend Brooks results to general hyperbolic singularities (cf. part I.), we obtain $g_{3}^{-}$. In this short note we cannot go into further details. Instead (for the moment) we will use Gao and Yau's result: $S^{3}$ admits a metric with $r(g)$. So we easily obtain $g_{3}^{-}$by taking connected sums $S^{3} \# \mathbb{R}^{3}=\mathbb{R}^{3}$.

The proof of case $n \geq 4$ is more complicated: The main step consists in the construction of a metric $g(n)$ on $S^{1} \times B_{2}(0) \subset S^{1} \times \mathbb{R}^{n-1}$ with $r(g(n))<0$ on $S^{1} \times B_{1}(0)$ and $g(n)=g_{S^{1}}+g_{E u c l}$. outside. To get this, one embeds $\overline{S^{1}} \times S^{1} \times B_{5}^{n-2}(0) \subset \overline{S^{1}} \times S^{1} \times \mathbb{R}^{n-2}$ into $S^{1} \times B_{1 / 2}(0)$ in some special way and uses Theorem 4 in dimension $n-1$ for $\overline{S^{1}} \times B_{5}^{n-2}(0)$ and $S^{1} \times B_{5}^{n-2}(0)$ to construct two metrics the combination of which yields (after some minor manipulations) $g(n)$. Finally, one embeds $S^{1} \times B_{3}(0)$ into $\mathbb{R}^{n}$ as tubular neighborhood of a large circle. Some not-too-hard deformation argument yields $g_{n}^{-}$.

To give an idea of part (2), we sketch the existence proof for negatively Ricci curved metrics in a very special case: the $n$-dimensional flat torus $T^{n}=$ $S^{1} \times \cdots \times S^{1}$, each factor with length $2 \pi \cdot 100$. For each $\left.\varrho \in\right] 0,1[$ there is a discrete subset $A_{\varrho} \subset T^{n}$ with

(i) $d(a, b)>5 \cdot \varrho$ for $a \neq b \in A_{\varrho}$;

(ii) $T^{n}=\bigcup_{a \in A_{e}} \overline{B_{5 \cdot \varrho}(a)}$;

(iii) $\#\left\{a \in A_{\varrho} \mid z \in B_{10 \cdot \varrho}(a)\right\} \leq c(n), c(n)$ independent of $z \in T^{n}$ and $\varrho \in] 0,1[$.

We define $g_{A_{\ell}}:=\varrho^{2} \cdot f_{a, \varrho}^{*}\left(g_{n}^{-}\right)$on $B_{2 \cdot \varrho}(a), a \in A_{\varrho}$, and $g_{A_{\ell}} \equiv g_{T^{n}}$ elsewhere with $f_{a, \varrho}: B_{2 \cdot \varrho}(a) \rightarrow \mathbb{R}^{n}, f_{a, \varrho} \equiv(1 / \varrho) \cdot I_{a} \circ \exp _{a}^{-1}$, where $I_{a}: T_{a} T^{n} \rightarrow \mathbb{R}^{n}$ is some linear isometry $\left(g_{A_{e}}\right.$ depends on the choice of $I_{a}$; for Theorem 2 one chooses $I_{f(a)}^{-1} \circ I_{a} \equiv D f_{a}$ for each $\left.f \in G \subset \operatorname{Diff}(M)\right)$. We are now ready to consider the following metric:

$$
g\left(A_{\varrho}, d, s\right):=\prod_{a \in A_{\varrho}} \exp \left(2 F_{d, s}^{\varrho} \cdot h_{\varrho}\left(10 \cdot \varrho-d\left(a, \mathrm{id}_{T^{n}}\right)\right)\right) \cdot g_{A_{\varrho}}
$$

where $F_{d, s}^{\varrho} \equiv s \cdot \exp \left(-d \cdot \varrho / \mathrm{id}_{\mathbb{R}}\right), h_{\varrho} \equiv h\left((1 / \varrho) \cdot \mathrm{id}_{\mathbb{R}}\right)$ with $h \in C^{\infty}(\mathbb{R},[0,1])$, $h \equiv 0$ on $\mathbb{R} \leq 1 / 2, h \equiv 1$ on $\mathbb{R}^{\geq 3 / 4}$, and $d(\cdot, \cdot)$ is the usual distance on $T^{n}$.

Then there are $d_{0}, s_{0}>0$ independent of $\varrho$ such that $r\left(g\left(A_{\varrho}, d, s\right)\right)<0$ for each $\left.d>d_{0}, s \in\right] 0, s_{0}$ [. Of course, for the flat torus $T^{n}, \varrho$ is a useless parameter, but think of the general case: A Besicovitch type argument gives similar coverings for each manifold $M^{n}$ (with $c(n)$ independent of $M^{n}$ ) if a suitable start metric is chosen. If $\varrho$ is chosen very small, the background metric on the given manifold appears quite "flat relative to $B_{\varrho}(a)$ ". Again, there are $\varrho, d, s$ such that an analogously defined metric $g\left(A_{\varrho}, d, s\right)$ is negatively Ricci curved, but $\varrho, d$, and $s$ are no longer independent.

\section{ACKNOWLEDGMENT}

This paper is an abstract of the author's doctoral thesis. The author thanks Professor Jürgen Jost for his friendly support. 


\section{REFERENCES}

[A] T. Aubin, Métriques riemanniennes et courbure, J. Differential Geom. 4 (1970), 383-424.

[BK] J. Bland and M. Kalka, Negative scalar curvature metrics on noncompact manifolds, Trans. Amer. Math. Soc. 316 (1989), 433-446.

[Bg] J. P. Bourguignon, Ricci curvature and Einstein metrics, Global Differential Geometry, Lecture Notes in Math., vol. 838, Springer, New York, 1981, pp. 42-63.

[Br1] R. Brooks, A construction of metrics of negative Ricci curvature, J. Differential Geom. 29 (1989), 85-94.

[G] L. Z Gao, The construction of negatively Ricci curved manifolds, Math. Ann. 271 (1985), 185-208.

[GY] L. Z. Gao and S. T. Yau, The existence of negatively Ricci curved on three manifolds, Invent. Math. 85 (1986), 637-652.

[Gr] M. Gromov, Structures métriques pour les variétés riemanniennes, Editions CEDIC, Paris, 1981.

[K] J. L. Kazdan, Prescribing the curvature of a Riemannian manifold, CBMS Regional Conf. Ser. in Math., vol. 57, Conf. Board Math. Sci., Washington, DC, 1985.

[T] W. Thurston, The geometry and topology of three manifolds, Princeton Lecture Notes, vol. 57, Princeton, NJ, 1980.

[Y] S. T. Yau, Seminar on differential geometry, problem section, Ann. of Math. Stud., vol. 102, Princeton Univ. Press, Princeton, NJ, 1982.

Ruhr-Universität Bochum, Mathematisches Institut, D-4630 Bochum, GeRmaNy 